\title{
The tangential velocity excess of the Milky Way satellites
}

\author{
Marius Cautun ${ }^{1 \star}$ and Carlos S. Frenk ${ }^{1}$ \\ 1 Department of Physics, Institute for Computational Cosmology, Durham University, South Road Durham DH1 3LE, UK
}

24 January 2018

\begin{abstract}
We estimate the systemic orbital kinematics of the Milky Way classical satellites and compare them with predictions from the $\Lambda$ cold dark matter $(\Lambda \mathrm{CDM})$ model derived from a semi-analytical galaxy formation model applied to high resolution cosmological N-body simulations. We find that the Galactic satellite system is atypical of $\Lambda \mathrm{CDM}$ systems. The subset of 10 Galactic satellites with proper motion measurements has a velocity anisotropy, $\beta=-2.2 \pm 0.4$, that lies in the $2.9 \%$ tail of the $\Lambda \mathrm{CDM}$ distribution. Individually, the Milky Way satellites have radial velocities that are lower than expected for their proper motions, with 9 out of the 10 having at most $20 \%$ of their orbital kinetic energy invested in radial motion. Such extreme values are expected in only $1.5 \%$ of $\Lambda$ CDM satellites systems. In the standard cosmological model, this tangential motion excess is unrelated to the existence of a Galactic "disc of satellites". We present theoretical predictions for larger satellite samples that may become available as more proper motion measurements are obtained.
\end{abstract}

Key words: Galaxy: halo - kinematics and dynamics - Local Group - cosmology: theory - dark matter

\section{INTRODUCTION}

Several predictions of the current cosmological paradigm the $\Lambda$ cold dark matter $(\Lambda \mathrm{CDM})$ model - agree with observations such as those of the temperature anisotropies of the cosmic microwave background radiation and galaxy clustering (e.g. see Frenk \& White 2012). Nonetheless, the model has been claimed to be in disagreement with some properties of the Local Group satellites. These claims include the observations that: there are far fewer dwarf galaxies than there are dark matter substructures (Klypin et al. 1999; Moore et al. 1999, a discrepancy misleadingly dubbed the "missing satellites" problem); that the internal structure of the most massive subhaloes is incompatible with that of known satellite galaxies (the "too-big-to-fail" problem; Boylan-Kolchin et al. 2011); and that a large fraction of satellites seem to rotate in a thin plane (the "planes of satellites" problem; Kroupa et al. 2005; Pawlowski \& Kroupa 2013; Ibata et al. 2013). The first two "problems" can be resolved by including realistic galaxy formation models (e.g. Sawala et al. 2016), but the latter is more challenging. Systematic studies of the Milky Way (MW) and M31 planes of satellites show that such configurations are uncommon, with only $\sim 10 \%$ of $\Lambda \mathrm{CDM}$ galactic-mass systems having more prominent planes than those in the Local Group (Cautun et al. 2015a,b).

In this letter, we compare the kinematics of the Galactic satellites with the predictions of $\Lambda \mathrm{CDM}$. We do so for the

^ E-mail : m.c.cautun@durham.ac.uk subset of 10 satellites that have HST proper motions (the 11 classical ones except Sextans). Previous studies have focused on two aspects of satellite kinematics: measuring the clustering of the orbital poles and reconstructing satellite orbits. The orbital poles are more clustered than an isotropic distribution, with the clustering being largest for a subset of eight of the 11 classical satellites (Pawlowski \& Kroupa 2013). Orbit reconstruction is more challenging since the outcome is sensitive to both the mass and the radial density profile of the MW halo (e.g. Lux et al. 2010; Barber et al. 2014), both of which are poorly constrained (Wang et al. 2015, and references therein). This leads to large uncertainties in the recovered orbits and, thus, a comparison with theoretical predictions is not very informative. To overcome such limitations, in this study we compare the velocity anisotropy parameter and the fraction of kinetic energy in radial motion between observations and theory. These two quantities are largely insensitive to the mass of the halo and to its radial density profile.

\section{DATA AND SIMULATIONS}

Our observational sample consists of the 10 bright Galactic satellites that have HST proper motions. These objects and the sources of their proper motion measurements are: Sagittarius - Pryor et al. (2010); LMC and SMC - Kallivayalil et al. (2013); Draco - Pryor et al. (2015), Ursa Minor - Piatek et al. (2005), Sculptor - Piatek et al. (2006), Carina Piatek et al. (2003), Fornax - Piatek et al. (2007), Leo II - 
Piatek et al. (2016); and Leo I - Sohn et al. (2013). We used satellite distances and heliocentric velocity values from the McConnachie (2012) compilation. To obtain the radial and tangential velocity components with respect to the Galactic Centre we followed the procedure described in Cautun et al. (2015b). We generate 1000 Monte Carlo realizations of the MW system in which we sample the satellite positions and proper motions from Gaussian distributions centred on the most likely values of each quantity and with dispersion equal to the uncertainties. These are transformed from heliocentric to Galactic coordinates, with the Monte Carlo realizations used to compute confidence intervals. The largest uncertainty is in the tangential velocities, with $1 \sigma$ errors varying from 20 to $55 \mathrm{~km} / \mathrm{s}$ (median value $40 \mathrm{~km} / \mathrm{s}$ ).

The theoretical model is based on the semi-analytic galaxy formation model of Henriques et al. (2015) applied to the Millennium II $\Lambda$ CDM dark matter cosmological simulation (Boylan-Kolchin et al. 2009), which has been rescaled to correspond to the Planck-1 values of the cosmological parameters (for details see Henriques et al. 2015). Our sample consists of haloes in the mass range, $M_{200}=(0.8-3.0) \times$ $10^{12} \mathrm{M}_{\odot}$, where $M_{200}$ is the mass enclosed by a spherical overdensity of 200 times the critical density. Our results are insensitive to the host halo mass, so we use a broad mass range motivated by the large uncertainties in the MW halo mass (Wang et al. 2015) and the advantages of having a large sample of MW analogues. We find 3672 such host haloes. We restrict the satellite selection to galaxies with a minimum stellar mass of $10^{5} \mathrm{M}_{\odot}$ found within a distance of $300 \mathrm{kpc}$ from the central galaxy. For each host, we select the 10 satellites with the largest stellar mass. In the case of the MW observations, we have proper motions for 10 satellites out of 12 objects brighter than $M_{\mathrm{V}}=-8.6$ (the classical satellites and Canes Venitici). To check for systematic biases, we constructed a second satellite catalogue by randomly selecting 10 out of the 12 objects with the largest stellar mass. We found that the two catalogues have the same satellite velocity distribution, so, for simplicity, we limit our analysis to the 10 brightest satellites.

We construct mock satellite catalogues to account for the uncertainties in the radial and tangential velocity components. We start by ranking the satellites according to their distance from the central galaxy. We do the same for the MW satellites. Then, the simulated satellites are assigned the errors corresponding to the MW satellite with the same rank, e.g. the innermost satellite in the simulation is linked to the MW innermost one. To model observational uncertainties, for every satellite we add to each velocity component a random value generated from a Gaussian distribution centred on zero with dispersion equal to the error reported for that velocity component. We repeat this procedure 10 times for each host, resulting in 36720 MW mocks.

\section{RESULTS}

The velocity anisotropy parameter, $\beta$, provides a simple measure of the kinematical properties of satellite galaxies. It is defined as:

$$
\beta=1-\frac{\sum_{i} V_{\mathrm{tan} ; \mathrm{i}}^{2}}{2 \sum_{i} V_{\mathrm{rad} ; \mathrm{i}}^{2}},
$$

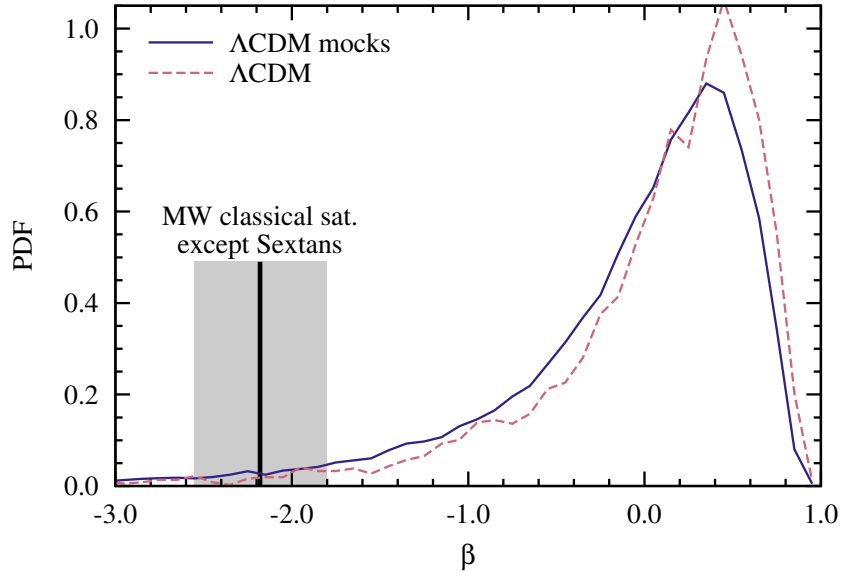

Figure 1. The distribution of the velocity anisotropy, $\beta$, for the 10 brightest satellites of MW-mass haloes. We show results for the cosmological simulation (dashed line) and for mock satellite catalogues that account for observational uncertainties (solid line). The vertical line shows the measured value, $\beta=-2.2 \pm 0.4$, for the MW satellites and the grey shaded region shows the $1 \sigma$ uncertainty interval. Only $2.9 \%$ of mock systems have a lower value of $\beta$ than the MW system.

where $V_{\text {rad; i }}$ and $V_{\text {tan; i }}$ denote the radial and tangential velocity components of satellite $i$ with respect to the central galaxy. The sum is over all the satellites associated with a host halo, which, in our case, is 10 . The $\beta$ parameter takes values in the range $-\infty$ to 1 , with $\beta<0, \beta=0$ and $\beta>0$ describing circularly-biased, isotropic and radially-biased orbits, respectively.

Figure 1 shows the distribution of $\beta$ values for the 10 brightest satellites of galactic mass haloes in our sample. We show the distribution for mock satellite catalogues and also for the original cosmological simulation (i.e. in the absence of velocity errors). In both cases, the satellite systems have radially-biased orbits, with a most likely value, $\beta \simeq 0.4$, but the $\beta$ distribution in the mock catalogues is slightly shifted towards lower values. The shift is due to the transverse velocity errors being an order of magnitude larger than the radial velocity errors. On average, this leads to an overestimation of $V_{\mathrm{tan} \text {; i }}^{2}$ by a larger amount than of $V_{\text {rad; i }}^{2}$, and thus a systematic reduction in $\beta$.

The Galactic satellites have $\beta=-2.2 \pm 0.4$, which means that they have tangentially-biased motions. This agrees with previous studies that, using fewer Galactic satellites with HST proper motions, also found a preference for tangential motions (e.g. Watkins et al. 2010; Pawlowski \& Kroupa 2013). The $\beta$ value of the Galactic satellites, marked with a vertical line in Figure 1, lies in the tail of the theoretical prediction, with only $2.9 \%$ of $\Lambda \mathrm{CDM}$ mock catalogues having an even more extreme value.

Figure 2 shows the distribution of tangential versus radial motion for individual satellites. We characterize this by the fraction of kinetic energy, $f_{\mathrm{E}}$; rad $=\frac{V_{\mathrm{rad}}^{2}}{V^{2}}$, along the radial direction. A satellite that, at a given moment, has a preferentially tangential motion corresponds to $f_{\mathrm{E} ; \mathrm{rad}}<\frac{1}{3}$, while a satellite that has a preferentially radial motion corresponds to $f_{\mathrm{E} ; \mathrm{rad}}>\frac{1}{3} . \Lambda \mathrm{CDM}$ predicts that at any moment $49 \%$ of the satellites have $f_{\mathrm{E} ; \mathrm{rad}}<\frac{1}{3}$, which increases to $52 \%$ for the Galactic mock satellite catalogues. 


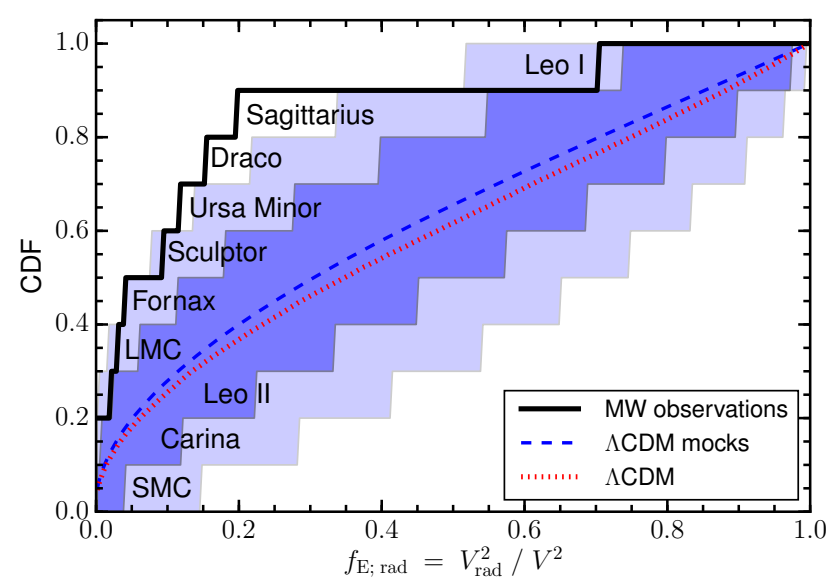

Figure 2. The distribution of the kinetic energy fraction in radial motion, $f_{\mathrm{E} ; \mathrm{rad}}=\frac{V_{\mathrm{rad}}^{2}}{V^{2}}$, for the 10 brightest satellites. The dashed line shows the median trend for the $\Lambda$ CDM Galactic mocks. The darker and lighter shaded regions show the $1-$ and $2-\sigma$ scatter regions. The distribution of MW satellites, which is shown by the solid line, is consistent with the mocks at the $1.5 \%$ level. We also show the median expectation in the absence of observational errors (dotted line).

The distribution of $f_{\mathrm{E} \text {; rad }}$ values for the Galactic satellites is dominated by tangential motions, with $f_{\mathrm{E} ; \text { rad }}<0.2$ for 9 out of the 10 satellites (thick solid line in Figure 2); on average $\Lambda \mathrm{CDM}$ predicts only 4 such objects. To quantify the significance of the disagreement between observations and theory we cannot just compute the fraction of mock catalogues that have 9 or more satellites with $f_{\mathrm{E} \text {; rad }}=0.2$ since this would be an a posteriori defined test that disregards the look elsewhere effect. This problem can be overcome by performing an extended Kolmogorov-Smirnov test that accounts for additional sources of scatter beyond just those due to Poisson statistics. We define the maximum difference between the cumulative distribution functions (CDF) of the data, $\mathrm{CDF}_{\text {data }}$, and of the mean for the mock catalogues, $\mathrm{CDF}_{\text {mean }}$, as:

$$
D=\max _{f_{\mathrm{E} ; \mathrm{rad}}}\left|\mathrm{CDF}_{\text {data }}\left(\mathrm{f}_{\mathrm{E} ; \mathrm{rad}}\right)-\mathrm{CDF}_{\text {mean }}\left(\mathrm{f}_{\mathrm{E} ; \mathrm{rad}}\right)\right| .
$$

The Galactic satellite system has $D_{\mathrm{MW}}=0.5$. For each mock catalogue, we compute $D$ given by Eq. (2) with the CDF of the data replaced by the $\mathrm{CDF}$ of the $f_{\mathrm{E}}$; rad values in that particular mock catalogue. The probability of obtaining a deviation as extreme as that observed in the data is given by the fraction of mock catalogues with $D$ values larger than $D_{\mathrm{Mw}}$. Only $1.5 \%$ of mock catalogues show a larger deviation than the data.

\section{DISCUSSION}

The $10 \mathrm{MW}$ satellites with measured proper motions have tangentially biased motions to an extent rarely found in $\Lambda \mathrm{CDM}$. Only $2.9 \%$ of $\Lambda \mathrm{CDM}$ systems have lower values of $\beta$ than the MW satellite system. Even fewer, $1.5 \%$, show deviations in the $\mathrm{CDF}$ of $f_{\mathrm{E}}$; rad that are as extreme as those measured for the MW. The two discrepancies are expressions of the same property, as may be seen in Figure 3. Selecting the $5 \%$ of mock catalogues with the lowest values of $\beta$ results in a distribution that is biased towards low $f_{\mathrm{E} \text {; rad values, }}$

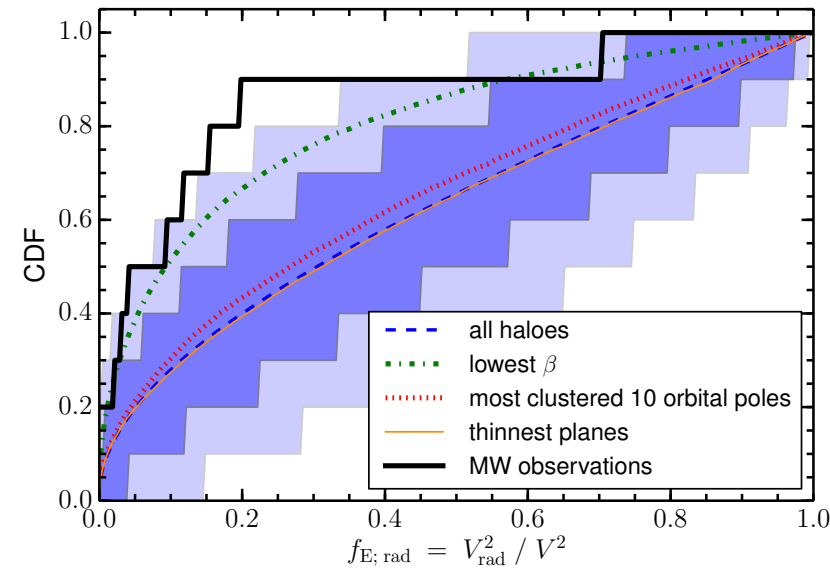

Figure 3. As Figure 2, but showing the median expectation for all $\Lambda$ CDM haloes (dashed line) and for subsets consisting of the $5 \%$ of haloes that have: the lowest $\beta$ values (dashed-dotted line), the most clustered satellite orbital poles (dotted line) and the thinnest satellite planes (solid thin line). The plot shows that low $\beta$ values are highly correlated with low $f_{\mathrm{E} ; \mathrm{rad}}$ values and thus both are expressions of the same phenomenon. The presence of a satellite plane or of coherent rotation has little effect on the $f_{\mathrm{E}}$; rad values and thus the two effects are largely independent.

similarly to that measured in the real data. In the following, we consider possible reasons behind the disparity between observations and theory. We focus on the test illustrated in Figure 2, i.e. the CDF of $f_{\mathrm{E} ; \mathrm{rad}}$, since that test shows the largest discrepancy and thus is the most constraining.

In Figure 3 we investigate whether the preference for tangential motions is somehow related to the Galactic "disc of satellites" (Lynden-Bell 1976; Kroupa et al. 2005; Libeskind et al. 2005). Very few haloes have satellite systems similar to that in the MW (Pawlowski et al. 2014), i.e. that are as thin and have highly clustered orbital poles, so, to have good statistics, we need to study each of these two aspects separately. First we select the $5 \%$ of mock satellite catalogues that have the thinnest planes of satellites. These are the systems with the smallest values of $c / a$, where $a$ and $c$ are respectively the major and minor axes of the inertia tensor of the satellite distribution. This subsample of haloes, shown with a thin solid line in Figure 3, has the same CDF of $f_{\mathrm{E}}$ rad values as the overall sample. Thus, the flattening of a satellite distribution is uncorrelated with its degree of tangential motion.

Of the 10 Galactic satellites with measured proper motions, 7 have orbital poles that are significantly clustered on the sky (Pawlowski \& Kroupa 2013). For each of our mock satellite systems we identify the set of $n$ satellites (out of 10) that have the most strongly clustered orbital poles, i.e. the smallest angular dispersion in the direction of the orbital poles (see Eq. 6 in Cautun et al. 2015b). For each value of $n$, we then select the $5 \%$ of haloes that have the most clustered orbital poles. These subsamples show a small preference for tangential motions compared to the full sample, with the excess being largest for $n=10$. The dotted line in Figure 3 shows the most extreme case, $n=10$. The MW data are consistent with this subsample at the $3.3 \%$ level. The weak correlation between preferentially tangential motions and clustering of the orbital poles may not be very relevant for the Galactic satellites. When considering the clustering 


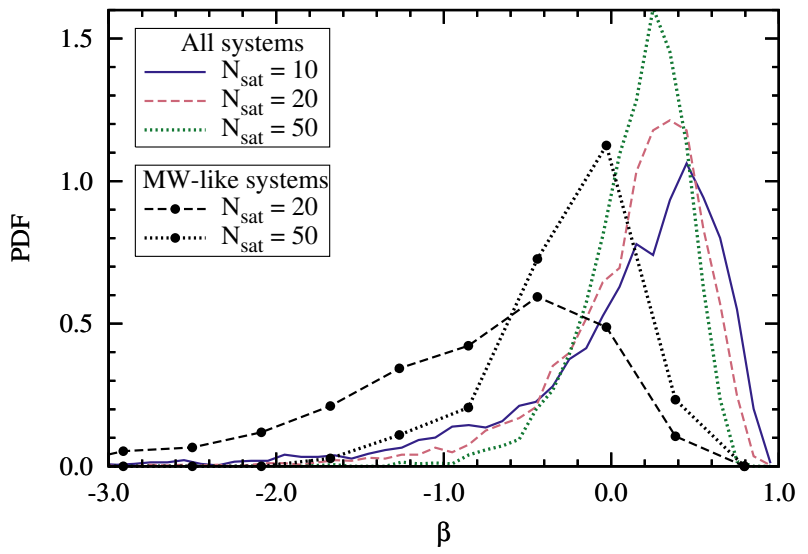

Figure 4. The distribution of velocity anisotropy, $\beta$, for the 10 (solid line), 20 (dashed line) and 50 (dotted line) brightest satellites of $\Lambda$ CDM Galactic-mass haloes. The lines with symbols show the same distribution but for haloes selected to resemble the MW, that is the $5 \%$ of haloes whose 10 brightest satellites have the lowest values of $\beta$. The results do not include velocity errors.

of all satellites (i.e. $n=10$ ), the MW is in the 15 th, not the 5 th, percentile of the distribution; mock catalogues corresponding to that percentile behave exactly like the full sample. The Galactic satellites are extreme for $n=7$, but the shift in the CDF in that case is much smaller than for the $n=10$ case shown in Figure 3. Thus, a strong clustering of the orbital poles of satellites is, at most, only weakly associated with an excess of tangential motion.

The LMC and the SMC are thought to have been accreted as a pair (e.g. Besla et al. 2012), so the two galaxies could have correlated orbital dynamics. This is unlikely to explain the tangential velocity excess of the Galactic satellites since group accretion is common in $\Lambda$ CDM: when studying the 11 brightest satellites, Wang et al. (2013) found accretion of satellite groups with 2 or more members in 5 out of their 6 haloes. Nonetheless, we tested for the effect of group accretion by excluding the SMC from the sample. We repeated the analysis for systems of 9 satellites and found only a small reduction in the difference between data and theory: the $9 \mathrm{MW}$ satellites lie in the $3.0 \%$ tail of the distribution for $\Lambda$ CDM.

Satellite proper motions are difficult to measure and could potentially be affected by unknown systematic errors. To reduce the Galactic tangential velocity excess to a $1 \sigma$ disagreement, the proper motion of each satellite would have to be overestimated by $45 \%$. Recently, Casetti-Dinescu \& Girard (2016) published a new ground-based proper motion measurement for Draco that is $\sim 6 \sigma$ discrepant from the Pryor et al. HST measurement. The ground-based measurement gives a much lower tangential velocity, $(90 \pm 16) \mathrm{km} / \mathrm{s}$, compared to the HST value, $(210 \pm 25) \mathrm{km} / \mathrm{s}$. Taking this value would ease the discrepancy between theory and observations, with only 8 out of the 10 Galactic satellites having $f_{\mathrm{E} ; \mathrm{rad}} \leqslant 0.2$, which would make the MW system a $9 \%$ outlier. It remains to be determined by future observations which of the two Draco proper motion measurements is correct and whether the HST measurements are affected by as yet unknown systematic errors. Two other concerns might be the limited resolution and the absence of baryonic effects in the cosmological simulation used here. We checked for these possibilities by analysing the COCO simulation (Hell-

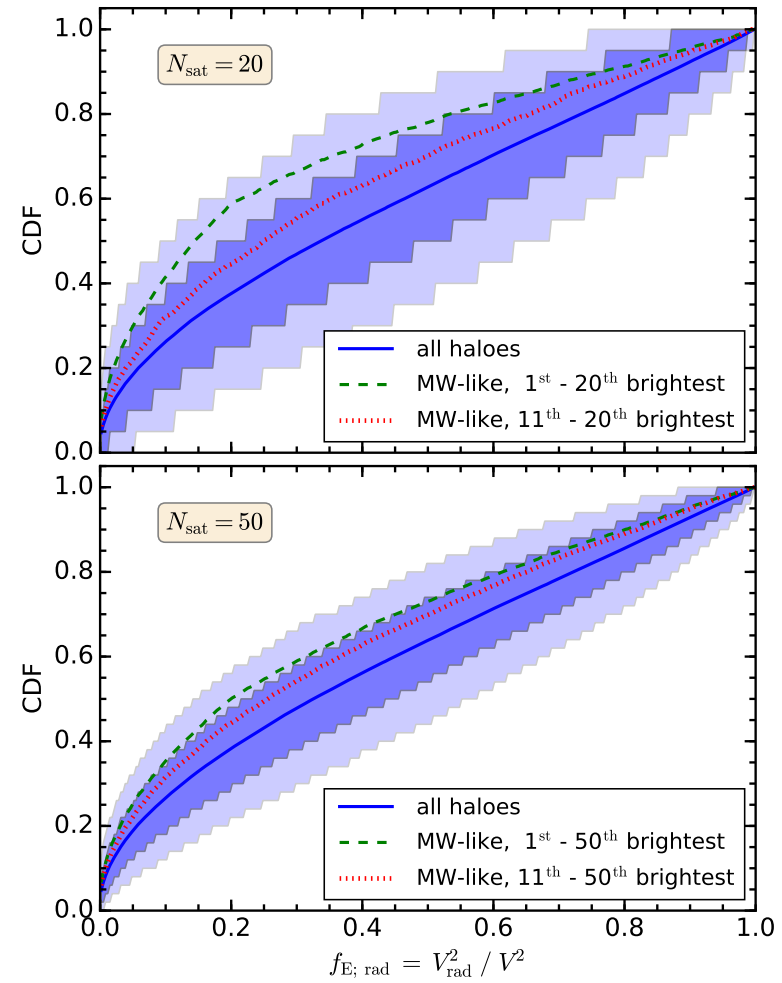

Figure 5. The distribution of the fraction of kinetic energy in radial motions, $f_{\mathrm{E}}$ rad, for the 20 (top panel) and 50 (bottom panel) brightest satellites of $\Lambda$ CDM Galactic-mass haloes. The darker and lighter shaded regions show the $1-$ and $2 \sigma$ scatter regions. The dashed line shows the expectation for haloes selected to resemble the MW, that is haloes for which at least 7 of the 10 brightest satellites have $f_{\mathrm{E}} ; \mathrm{rad} \leqslant 0.2$ (which corresponds to $\sim 5 \%$ of the population). The dotted line shows the expectation for the MW-like systems when excluding the 10 brightest satellites, i.e. when considering only the $11^{\text {th }}-20^{\text {th }}$ or the $11^{\text {th }}-50^{\text {th }}$ brightest samples. The results do not include velocity errors.

wing et al. 2016, which has 100 times better mass resolution) and the APOSTLE Local Group simulations (Sawala et al. 2016, which include realistic baryonic physics). We found good agreement between the results of these simulations and those of the one used in this study.

The fraction of kinetic energy invested in radial motions, $f_{\mathrm{E} ; \mathrm{rad}}$, depends on the position of the satellite along its orbit, being smallest at pericentre and apocentre. The low $f_{\mathrm{E} ; \mathrm{rad}}$ value found for the Galactic satellites could be interpreted as implying that 9 of the 10 satellites are close to either pericentre or apocentre to a larger extent than is normally found in $\Lambda \mathrm{CDM}$. The $f_{\mathrm{E}}$ rad value also depends on the orbital ellipticity, being smaller for circularly biased obits. Thus, the discrepancy between data and theory could alternatively indicate that the Galactic satellites have orbits that are, on average, closer to circular than is typical in $\Lambda$ CDM. This would mean that MW halo mass estimates based on satellite orbits (e.g. Barber et al. 2014) are biased low.

More observations are required to decide which, if any, of the above explanations is correct, or, alternatively, if the excess of tangential motions is an indication of new physics in the dark sector. There are two main directions in which this analysis can be extended: measuring proper motions for fainter Galactic satellites or performing similar tests for ex- 
ternal galaxies. Figures 4 and 5 show theoretical predictions for the expected behaviour of $\beta$ and $f_{\mathrm{E}}$; rad as proper motion measurements become available for a larger number of satellites. The Gaia mission will reduce the uncertainties in the proper motions of several of the classical satellites and should obtain new measurements for fainter objects, especially for those within $\sim 100 \mathrm{kpc}$ from the Sun (Wilkinson \& Evans 1999). The proper motions of more distant Galactic satellites and of those in M31 could be measured by a dedicated multi-year HST programme and by follow-up with JWST and WFIRST (Kallivayalil et al. 2015).

Figure 4 shows that the velocity anisotropy, $\beta$, decreases as fainter satellites are included in the sample, with the typical value varying from $\beta=0.45$ for the 10 brightest satellites to $\beta=0.25$ for the 50 brightest satellites. The distribution of $\beta$ becomes more peaked and narrower for larger satellite samples. To make predictions constrained by the already existing data for the MW, where the 10 brightest satellites have very low $\beta$ values, we select the $5 \%$ of haloes whose 10 brightest satellites have the lowest velocity anisotropy $(\beta \leqslant-1.3)$. The velocity anisotropy of the 20 or 50 brightest satellites remains biased low for these systems relative to the full sample of haloes.

In Figure 5 we show the CDF of the kinetic energy fraction in radial motion for the 20 and 50 brightest satellites. While the median trend hardly changes, the scatter is much reduced as the number of satellites increases. The dashed curves show the expected behaviour for $\Lambda$ CDM systems chosen to be similar to the MW, that is in haloes for which at least 7 of the 10 brightest satellites have $f_{\mathrm{E} ; \mathrm{rad}} \leqslant 0.2$. These MW-like systems show systematically larger tangential motions even when excluding the 10 brightest satellites, which were used in the first place to select the sample.

\section{SUMMARY}

We have found that the bright satellites of the MW have larger tangential orbital motions than expected from $\Lambda \mathrm{CDM}$ cosmological simulations. This excess is most clearly manifest in the fraction of kinetic energy along the radial direction, $f_{\mathrm{E} ; \mathrm{rad}}$, with 9 of the $10 \mathrm{MW}$ satellites with HST proper motion measurements having $f_{\mathrm{E} ; \mathrm{rad}}<0.2$. Such extreme values are found in at most $1.5 \%$ of $\Lambda \mathrm{CDM}$ galactic satellite systems. This conclusion, of course, relies on the accuracy of current HST proper motion measurements, which has been called into question by a recent measurement of Draco using ground-based data. In $\Lambda \mathrm{CDM}$, the tangential motion excess is unrelated to the existence of a Galactic "disc of satellites" and cannot be explained by the accretion of satellite groups. More satellites with measured proper motions are required to check if the observed excess is merely an indication that the MW is atypical or if it poses a problem for the $\Lambda \mathrm{CDM}$ model.

\section{ACKNOWLEDGEMENTS}

We thank Andrew Cooper, Alis Deason, Mark Gieles and Till Sawala for helpful discussions and comments. This work was supported in part by ERC Advanced Investigator grant COSMIWAY [grant number GA 267291] and the Science and Technology Facilities Council [grant number ST/F001166/1, ST/I00162X/1]. This work used the
DiRAC Data Centric system at Durham University, operated by ICC on behalf of the STFC DiRAC HPC Facility (www.dirac.ac.uk). This equipment was funded by BIS National E-infrastructure capital grant ST/K00042X/1, STFC capital grant ST/H008519/1, and STFC DiRAC Operations grant ST/K003267/1 and Durham University. DiRAC is part of the National E-Infrastructure. Data from the Millennium-II simulation is available on a relational database accessible from http://gavo.mpa-garching.mpg. de/Millennium/ .

\section{REFERENCES}

Barber C., Starkenburg E., Navarro J. F., McConnachie A. W., Fattahi A., 2014, MNRAS, 437, 959

Besla G., Kallivayalil N., Hernquist L., van der Marel R. P., Cox T. J., Kereš D., 2012, MNRAS, 421, 2109

Boylan-Kolchin M., Springel V., White S. D. M., Jenkins A., Lemson G., 2009, MNRAS, 398, 1150

Boylan-Kolchin M., Bullock J. S., Kaplinghat M., 2011, MNRAS, 415, L40

Casetti-Dinescu D. I., Girard T. M., 2016, MNRAS, 461, 271

Cautun M., Wang W., Frenk C. S., Sawala T., 2015a, MNRAS, 449,2576

Cautun M., Bose S., Frenk C. S., Guo Q., Han J., Hellwing W. A., Sawala T., Wang W., 2015b, MNRAS, 452, 3838

Frenk C. S., White S. D. M., 2012, Annalen der Physik, 524, 507

Hellwing W. A., Frenk C. S., Cautun M., Bose S., Helly J., Jenkins A., Sawala T., Cytowski M., 2016, MNRAS, 457, 3492

Henriques B. M. B., White S. D. M., Thomas P. A., Angulo R., Guo Q., Lemson G., Springel V., Overzier R., 2015, MNRAS, 451,2663

Ibata R. A., et al., 2013, Nature, 493, 62

Kallivayalil N., van der Marel R. P., Besla G., Anderson J., Alcock C., 2013, ApJ, 764, 161

Kallivayalil N., et al., 2015, preprint, (arXiv:1503.01785)

Klypin A., Kravtsov A. V., Valenzuela O., Prada F., 1999, ApJ, 522, 82

Kroupa P., Theis C., Boily C. M., 2005, A\&A, 431, 517

Libeskind N. I., Frenk C. S., Cole S., Helly J. C., Jenkins A., Navarro J. F., Power C., 2005, MNRAS, 363, 146

Lux H., Read J. I., Lake G., 2010, MNRAS, 406, 2312

Lynden-Bell D., 1976, MNRAS, 174, 695

McConnachie A. W., 2012, AJ, 144, 4

Moore B., Ghigna S., Governato F., Lake G., Quinn T., Stadel J., Tozzi P., 1999, ApJ, 524, L19

Pawlowski M. S., Kroupa P., 2013, MNRAS, 435, 2116

Pawlowski M. S., et al., 2014, MNRAS, 442, 2362

Piatek S., Pryor C., Olszewski E. W., Harris H. C., Mateo M., Minniti D., Tinney C. G., 2003, AJ, 126, 2346

Piatek S., Pryor C., Bristow P., Olszewski E. W., Harris H. C., Mateo M., Minniti D., Tinney C. G., 2005, AJ, 130, 95

Piatek S., Pryor C., Bristow P., Olszewski E. W., Harris H. C., Mateo M., Minniti D., Tinney C. G., 2006, AJ, 131, 1445

Piatek S., Pryor C., Bristow P., Olszewski E. W., Harris H. C., Mateo M., Minniti D., Tinney C. G., 2007, AJ, 133, 818

Piatek S., Pryor C., Olszewski E. W., 2016, AJ, 152, 166

Pryor C., Piatek S., Olszewski E. W., 2010, AJ, 139, 839

Pryor C., Piatek S., Olszewski E. W., 2015, AJ, 149, 42

Sawala T., et al., 2016, MNRAS, 457, 1931

Sohn S. T., Besla G., van der Marel R. P., Boylan-Kolchin M., Majewski S. R., Bullock J. S., 2013, ApJ, 768, 139

Wang J., Frenk C. S., Cooper A. P., 2013, MNRAS, 429, 1502

Wang W., Han J., Cooper A. P., Cole S., Frenk C., Lowing B., 2015, MNRAS, 453, 377

Watkins L. L., Evans N. W., An J. H., 2010, MNRAS, 406, 264

Wilkinson M. I., Evans N. W., 1999, MNRAS, 310, 645 